\title{
Flow Calculation in Vertical Cavity with Free Surface and Bottom Opening to Water Stream
}

\author{
by Kazuhiro Fukuda*, Member
}

Yutaro Yoshii**,

\begin{abstract}
Summary
The flow in a two-dimensional cavity with a free surface and a bottom opening on a channel stream is numerically calculated. The calculated results are compared with experimental data measured in the vertical openings of ship models. The calculations are performed using GSM A C-FEM. The arbitrary Lagrangian-Eulerian formulation is applied to deal with the free surface motion. To test the algorithm used in the present calculation, three cases of the fluid problem are examined: normal cavity flow, free oscillation of water in a tank, and the run up height of a solitary wave on a tank wall. In the three cases, the calculation results show good agreements with previously published data. Using the present calculation algorithm, the flow in a cavity with a free surface and a bottom opening to a channel flow is calculated and the following conclusions are obtained: (1) Vertical oscillations of the free surface in the cavities are observed in the numerical calculation when there are two vertical cavities with the free surface on the upper wall of a channel flow. (2) The stream velocities of the free surface motion in the numerical calculation are the same as those of the experimental results measured in the vertical opening of ship models at the dimensionless speed, $2 \mathrm{U} /(\mathrm{L} \omega$ o) or $1 /(\pi \mathrm{St})$.
\end{abstract}

Key Words: Cavity Flow, F ree Surface, GSM AC-FEM, ALE M ethod, M oon pool, Sloshing problem

\section{INTRODUCTION}

$M$ any researchers have studied the cavity flow problem in order to verify the validity of their numerical calculation techniques. However, a cavity that consists of a free surface on the top and flow on the bottom has not been studied. Cavities with a free surface occur in certain kinds of ships that have vertical holes in their hulls. One such ship is a drilling vessel that has a vertical hole called a moonpool ${ }^{1), 22}$. It is used for lowering a boring drill-pipe to the sea bottom. One of the present authors, K.Fukuda, conducted model tests in a towing water tank and reported ${ }^{1)}$ that the water in a moonpool moves up and down, which increases the ship resistance at certain ship speeds.

This present paper presents numerical calculations of the water motion in a two-dimensional vertical cavity with a free surface on the top and a current on the bottom. The calculation is performed using the GSMAC-FEM ${ }^{3}$ ) (Genaralized Simplified $M$ arker and Cell - Finite Element $M$ ethod) for the A LE (arbitrary Lagrangian - Eulerian) formulation.

In order to verify the validity of this calculation method, calculations are performed for three practical cases; normal cavity flow, free oscillation of a liquid in a tank, and the run-up height of a solitary wave on a tank wall. The results obtained are compared with previously published calculation results.

The calculation for the flow in the cavity with a free surface is performed by inserting two cavities in the channel upward wall. Computed results for the amplitude and frequency of the surface motion are compared with previously published experimental results of the vertical opening of the ship models.

* Nara National College of Technology

** Nagoya University, Graduate School of Information Science (Researched at Advanced Engineering Course of Nara N ational College of Technology)

Received 21stApr. 2009

Read at the spring meeting

\section{NUMERICAL CALCULATION}

2.1 Goveming Equations

The ALE formulation is applied to calculate the motion of the free surface. The ALE equations for two-dimensional laminar flow of an incompressible liquid are as follows:

$$
\begin{aligned}
& \frac{\partial u_{i}}{\partial t}+\left(u_{j}-\hat{u}_{j}\right) \frac{\partial u_{i}}{\partial x_{j}}=-\frac{\partial P}{\partial x_{i}}+\frac{\partial \tau_{i j}}{\partial x_{j}} \\
& P=p+x_{2} / F^{2} \\
& \tau_{i j}=\frac{1}{R e}\left(\frac{\partial u_{i}}{\partial x_{j}}+\frac{\partial u_{j}}{\partial x_{i}}\right) \\
& R e=\frac{\rho U L}{\mu} \\
& F r=\frac{U}{\sqrt{g L}}
\end{aligned}
$$

where $\mathrm{U}_{\mathrm{j}}$ is the velocity components in $\mathbf{X}_{\mathrm{j}}$ direction, and $\hat{U}_{j}$ is the grid velocity. $P$ is the potential, which is the sum of the pressure $p$ and the gravitational potential of the function of Froude number $\mathrm{Fr} . \quad \tau_{\mathrm{ij}}$ is the stress tensor. These variables are converted to non-dimensional forms using the length $L$, speed $\mathrm{U}$ and density $\rho . \mathrm{In}$ the present paper, the Einstein summation rule is applied to the same subscripts.

\subsection{Equation of free surface}

The free surface form is determined from the fact that the surface moves with the liquid speed.

$$
\frac{\partial \mathrm{H}}{\partial \mathrm{t}}+\left(\mathrm{u}_{1}-\hat{\mathrm{u}}_{1}\right) \frac{\partial \mathrm{H}}{\partial \mathrm{x}_{1}}=\mathrm{u}_{2}
$$

where $\mathrm{H}$ is the free surface height measured from the channel bottom. In this study, the distortion of the free surface is assumed to be small and the surface tension is neglected. The 
boundary condition on the free surface is given by

$$
p-n_{i} \tau_{i j} n_{j}=p_{0}
$$

where $n_{i}$ is the unit normal vector to the free surface, $p_{0}$ is the atmospheric pressure( its value is taken to be zero).

\subsection{GSMAC-FEM method}

(1) Time discretization

The calculation is performed using the GSMAC-FEM method described in Ref. 3). In this method, the governing equation, Eq.(1), is discretized into a semi-implicit formulation, namely velocity is explicitly discretized and pressure is implicitly discretized. A balancing tensor diffusivity (BTD) term is added in order to increase the accuracy in time and stability.

The procedure in one computational time step $\Delta$ t consists of following four steps.

(Predictor step)

$$
\begin{aligned}
\frac{\tilde{u}_{i}-u_{i}^{n}}{\Delta t} & +\left(u_{j}^{n}-\hat{u}_{j}^{n}\right) \frac{\partial u_{i}^{n}}{\partial x_{j}}=-\frac{\partial P^{n}}{\partial x_{i}} \\
+ & \frac{\partial \tau_{i j}^{n}}{\partial x_{j}}+\frac{\partial}{\partial x_{j}}\left(\frac{\Delta t}{2} u_{j}^{n} u_{k}^{n} \frac{\partial u_{i}^{n}}{\partial x_{k}}\right)
\end{aligned}
$$

(Poisson Equation step)

$$
\frac{\partial^{2} \delta P}{\partial x_{j} \partial x_{j}}=\frac{1}{\Delta t} \frac{\partial \tilde{u}_{j}}{\partial x_{j}}
$$

(Correction step)

$$
\begin{aligned}
& \frac{u_{i}^{n+1}-\tilde{u_{i}}}{\Delta t}=-\frac{\partial \delta P}{\partial x_{i}} \\
& \mathrm{P}^{\mathrm{n}+1}=\mathrm{P}^{\mathrm{n}}+\delta \mathrm{P}
\end{aligned}
$$

(J udgment step)

$$
\frac{\partial \tilde{u}_{j}}{\partial x_{j}}<\varepsilon
$$

where $\tilde{u}_{i}$ is the predictor of velocity and $\delta P$ is the modified potential including the pressure. The superscript $n$ means the computational time step number, $\mathrm{t}=\mathrm{n} \Delta \mathrm{t}$. The Poisson equation for $\delta P$ is solved by the simultaneous relaxation method based on the Newton-Raphson scheme. $u_{i}^{n+1}$ and $P^{n+1}$ are obtained from Eqs. (10) and (11), respectively. However, the continuity equation is not satisfied at this iterative step. Because Eq.(9) is solved by the N ewton-Raphson scheme with the approximation of lumped mass matrix. So these iteration steps must be repeated using the next iteration with $\tilde{u}=u_{i}^{n+1}$ and $p^{n}=p^{n+1}$, until the convergence criterion of $E q .(12)$ is satisfied. After this criterion is satisfied, time advances by one step $\Delta \mathrm{t}$.

\section{(2) S patial discretization by FEM}

The spatial discretizaion is made with quadrilateral elements by Galerkin FEM. The velocity in a cell, $u_{i}$, is interpolated by the shape function $\mathrm{N}_{\mathrm{a}}$ with the velocity at the nodal point velocity $\mathrm{u}_{\mathrm{ia}}$.

$$
\begin{aligned}
& \mathrm{u}_{\mathrm{i}}=\mathrm{N}_{\mathrm{k}} \mathrm{u}_{\mathrm{ik}} \\
& \mathrm{N}_{\mathrm{a}}=\frac{1}{4}\left(1+\xi_{\mathrm{a}} \xi\right)\left(1+\eta_{\mathrm{a}} \eta\right) \quad \text { (no sum on } \mathrm{a} \text { ) }
\end{aligned}
$$

where $\xi_{\mathrm{a}}=+1$ or -1 , and $\eta_{\mathrm{a}}=+1$ or -1 , which depend on the subscript a.

Eqs.(8)-(12) are discretized as follows:

(Predictor step)

$$
\begin{gathered}
\bar{M}_{a b} \frac{\tilde{u}_{i b}-u_{i b}^{n}}{\Delta t}=-\left[C_{a b}+\frac{1}{R e} D_{a b}^{k k}+\frac{\Delta t}{2} B_{a b}\right] u_{i b}^{n} \\
+G_{a}^{i} P_{e}^{n}-\frac{1}{R e} D_{a b}^{i j} u_{j b}^{n}+S_{a}^{i}
\end{gathered}
$$

( Poisson equation step)

$$
\begin{aligned}
& \delta P_{e}=\frac{G_{b}^{j} \tilde{u}_{j b}}{\Delta t}\left(\sum_{k=1}^{4} \frac{G_{k}^{j} G_{k}^{j}}{\bar{m}}\right)^{-1} \\
& \bar{m}={\overline{M_{11}}}^{A}+{\overline{M_{22}}}^{B}+{\overline{M_{33}}}^{C}+{\overline{M_{44}}}^{D}
\end{aligned}
$$

(Correction step)

$$
\begin{aligned}
& \bar{M}_{a b} \frac{u_{i b}^{n+1}-\tilde{u}_{i b}}{\Delta t}=G_{a}^{i} \delta P_{e}-\oint_{\Gamma_{e}} N_{a} \delta P_{e} n_{i} d \Gamma \\
& P_{e}^{n+1}=P_{e}^{n}+\delta P_{e}
\end{aligned}
$$

(J udgment step)

$$
\frac{G_{b}^{j} \tilde{u}_{j b}}{A^{e}}<\varepsilon
$$

Coefficient matrices in Eqs.(15)-(20) are as follows.

$$
\begin{aligned}
& \mathrm{M}_{\mathrm{ab}}=\int_{\Omega^{\mathrm{e}}} \mathrm{N}_{\mathrm{a}} \mathrm{N}_{\mathrm{b}} \mathrm{d} \Omega \\
& \mathrm{G}_{\mathrm{a}}^{\mathrm{i}}=\int_{\Omega^{\mathrm{e}}} \frac{\partial \mathrm{N}_{\mathrm{a}}}{\partial \mathrm{x}_{\mathrm{i}}} \mathrm{d} \Omega \\
& \mathrm{C}_{\mathrm{ab}}=\left(\mathrm{u}_{\mathrm{j}}^{\mathrm{e}}-\hat{\mathrm{u}}_{\mathrm{j}}^{\mathrm{e}}\right) \int_{\Omega^{\mathrm{e}}} \mathrm{N}_{\mathrm{a}} \frac{\partial \mathrm{N}_{b}}{\partial \mathrm{x}_{\mathrm{j}}} \mathrm{d} \Omega \\
& \mathrm{D}_{\mathrm{ab}}^{\mathrm{ij}}=\int_{\Omega^{\mathrm{e}}} \frac{\partial \mathrm{N}_{\mathrm{a}}}{\partial \mathrm{x}_{\mathrm{j}}} \frac{\partial \mathrm{N}_{b}}{\partial \mathrm{x}_{\mathrm{i}}} \mathrm{d} \Omega \\
& \mathrm{B}_{\mathrm{ab}}=\mathrm{u}_{\mathrm{i}}^{\mathrm{e}} \mathrm{u}_{\mathrm{j}}^{\mathrm{e}} \mathrm{D}_{\mathrm{ab}}^{\mathrm{ij}} \\
& \mathrm{S}_{\mathrm{a}}^{\mathrm{i}}=\oint_{\Gamma^{\mathrm{e}}} \mathrm{N}_{\mathrm{a}}\left(-\mathrm{P}^{\mathrm{n}} \delta_{\mathrm{ij}}+\tau_{\mathrm{ij}}^{\mathrm{n}}\right)_{\mathrm{j}} \mathrm{d} \Gamma \\
& \mathrm{u}_{\mathrm{i}}^{\mathrm{e}}=\frac{1}{\mathrm{~A}^{\mathrm{e}}} \int_{\Omega^{\mathrm{e}}} \mathrm{N}_{\mathrm{k}} \mathrm{u}_{\mathrm{ik}} \mathrm{d} \Omega
\end{aligned}
$$

where the subscripts $a$ and $b$ denote the local node number (from 1 to 4 ) and $i$ and $j$ denote coordinate and velocity numbers (from 1 to 2 ). The superscript e is the mean value on the element $\Omega$. The velocity is interpolated with a set of shape function $N_{a}$ on the element $\Omega$. The potential including the pressure, $\mathrm{P}_{\mathrm{e}}$, is assumed to be constant on $\Omega . \overline{\mathrm{M}}_{\mathrm{ab}}$ is the lumped mass matrix which is obtained by summing up the values across each row of the consistent mass matrix $M_{a b}$ $=\int_{\Omega^{e}} N_{a} N_{b} d \Omega$ and placing the summed results in the diagonal. $m$ in $E q .(17)$ is the summation of the lamped mass matrices from $A$ to $D$ elements which surround the local node point. Fig. 1 shows the $A \sim D$ elements and their nodal point numbers. $u_{i}^{e}$ is the mean value of $u_{i}^{n}$, and $A^{e}$ is the cell area. $\Gamma^{e}$ denotes the interface of the element and $n_{i}$ is the normal vector to the interface. $\delta_{\mathrm{ab}}$ is the K ronecker delta.

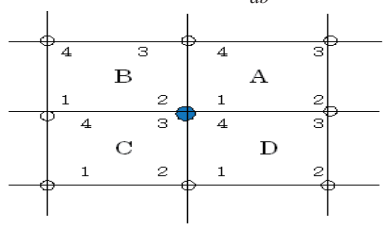

Fig. 1 Nodal point numbers of surrounding a point 
The boundary condition on the free surface, Eq.(7), is substituted into Eq.(26) of the free surface elements. When the distortion of the free surface is assumed to be small, Eq.(26) is approximated in the following form on the free surface.

$$
\mathrm{S}_{\mathrm{a}}^{\mathrm{i}}=\oint_{\Gamma} \mathrm{N}_{\mathrm{a}}\left(-\mathrm{x}_{2} / \mathrm{Fr}^{2}\right) \delta_{\mathrm{ij}} \mathrm{n}_{\mathrm{j}} \mathrm{d} \Gamma
$$

On the Direchlet boundary condition parts, for example on the wall, Eq.(26) is 0 , because we can assume that the shape function $\mathrm{N}_{a}$ of the weight function is 0 .

\subsection{Discretization of free surface by FEM}

The free surface equation, Eq.(6), is discretized on time marching to Eq.(29). This equation also includes the BTD term. $\hat{\mathrm{u}}_{1}$ is taken to be zero because the distortion of the free surface is assumed to be small and $X_{1}$ coordinate is not moved.

$$
\begin{array}{r}
\frac{\mathrm{H}^{\mathrm{n}+1}-\mathrm{H}^{\mathrm{n}}}{\Delta \mathrm{t}}=-\mathrm{u}_{1}^{\mathrm{n}} \frac{\partial \mathrm{H}^{\mathrm{n}}}{\partial \mathrm{x}}+\mathrm{u}_{2}^{\mathrm{n}} \\
+\frac{\partial}{\partial \mathrm{x}}\left(\frac{\Delta \mathrm{t}}{2} \mathrm{u}_{1}^{\mathrm{n}} \mathrm{u}_{1}^{\mathrm{n}} \frac{\partial \mathrm{H}^{\mathrm{n}}}{\partial \mathrm{x}}\right)
\end{array}
$$

Eq.(29) is discretized in space by one-dimensional Galerkin FEM as follows:

$$
\begin{aligned}
\bar{M}_{\mathrm{ab}} & \frac{\mathrm{H}_{\mathrm{b}}^{\mathrm{n}+1}-\mathrm{H}_{\mathrm{b}}^{\mathrm{n}}}{\Delta \mathrm{t}}= \\
& -\left[\mathrm{C}_{\mathrm{ab}}+\frac{\Delta \mathrm{t}}{2} \mathrm{~B}_{\mathrm{ab}}\right] \mathrm{H}_{\mathrm{b}}^{\mathrm{n}}+\frac{\Delta \mathrm{x}}{2} \mathrm{u}_{2}^{\mathrm{e}} \\
\overline{\mathrm{M}}_{\mathrm{ab}} & =\frac{\Delta \mathrm{x}}{2} \delta_{\mathrm{ab}} \\
{\left[\mathrm{C}_{\mathrm{ab}}\right] } & =\frac{\mathrm{u}_{1}^{\mathrm{e}}}{2}\left[\begin{array}{cc}
-1 & 1 \\
-1 & 1
\end{array}\right] \\
{\left[\mathrm{B}_{\mathrm{ab}}\right] } & =\frac{\left(\mathrm{u}_{1}^{\mathrm{e}}\right)^{2}}{\Delta \mathrm{x}}\left[\begin{array}{cc}
1 & -1 \\
-1 & 1
\end{array}\right]
\end{aligned}
$$

where $\overline{\mathrm{M}}_{\mathrm{ab}}$ is the lumped mass matrix, and $\mathrm{u}_{1}^{\mathrm{e}}$ and $\mathrm{u}_{2}^{\mathrm{e}}$ are the mean values of the velocity in the cell.

\section{VALIDITY of PRESENT SCHEME}

In order to assess the validity of the present calculation algorithm, the following three cases were calculated and results obtained were compared with previously published data.

\subsection{Closed cavity flow}

There are a lot of calculation results for a closed cavity in which one wall moves at a constant speed. Boundary conditions of all walls are non-slip conditions. Fig. 2 shows the calculated velocity in the cavity ( $30 \times 30$ grid) and velocity distributions at the horizontal and vertical center lines in the cavity and compares these results with the data of Hashimoto ${ }^{3)}$ and $\mathrm{Ghia}^{4)}$.
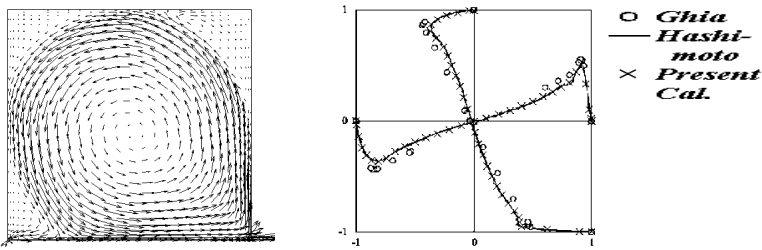

Fig. 2 Computed flow velocity in cavity $(R e=5000)$

3.2 Free oscillation of a liquid in a tank

The free oscillation of a liquid in a tank with a unit height and a unit width is calculated and the results are compared with Ramaswamy data ${ }^{5)}$. The initial profile of the free surface is given by $\mathrm{Eq} .(34)$.

$$
h=1+h_{6} \sin \pi\left(1-x_{1}\right)
$$

where $x_{1}$ is the horizontal axis $\left(-0.5 \leq x_{1} \leq 0.5\right)$ and $h_{0}$ is the initial amplitude $\left(h_{0}=0.01\right)$. The slip boundary conditions exist on the side and bottom walls of the tank. The atmospheric pressure is set to be zero on the free surface. The calculations are performed with and without water viscosity.

Fig.3 shows the time histories of the free surface heights at the left and right walls. The TIME in Fig.3 means the dimensionless time. The upper graphs are for a inviscid liquid and the lower graphs are for a viscous liquid at $R e=100$. The first two graphs on the left-hand side are taken from R ef. 5).

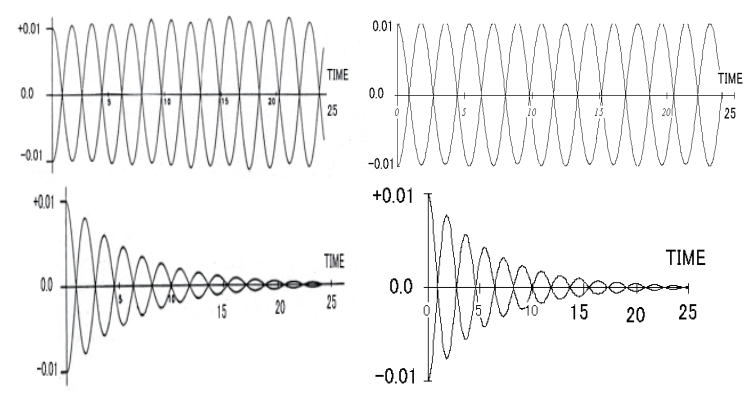

$\begin{array}{ll}\text { (a) Ramaswamy result } & \text { (b) Present Calculation }\end{array}$

Fig.3 Time histories of the free surface heights at both walls (upper: without viscous effect, lower: with viscous effect)

\subsection{Run-up height of a solitary wave}

A nalysis of the propagation of a solitary wave ${ }^{5), 6)}$ is a good example for assessing the validity of the present calculation method. Ramaswamy calculated a solitary wave propagation in a two dimensional tank of which depth is 1.0 unit, $\mathrm{d}$, and width is 16.0 units, $16 \mathrm{~d}$.

The initial height form and velocity distribution of the solitary wave used in the calculation are given in Ref.5). The theoretical run-up height $\mathrm{R}$ at the wall is given as Eq.(35) by Ramaswamy ${ }^{5}$.

$$
\frac{\mathrm{R}}{\mathrm{d}}=2 \frac{\mathrm{h}_{0}}{\mathrm{~d}}+\frac{1}{2}\left(\frac{\mathrm{h}_{0}}{\mathrm{~d}}\right)^{2}
$$

where $h_{0}$ is the initial solitary wave height. Fig.4 shows the pattern of the solitary wave propagation at each dimensionless 
time step for the inviscid liquid. Fig.5 shows a comparison of the calculated results for the run-up height $\mathrm{R} / \mathrm{d}$ with the theoretical one. The horizontal axis represents the initial solitary wave height $h_{0} / d$. The calculations are performed for both without viscous effect $(R e=\infty)$ and with viscous effect $(\mathrm{Re}=100)$. Fig.5 shows that present computed results agree well with the theoretical values.

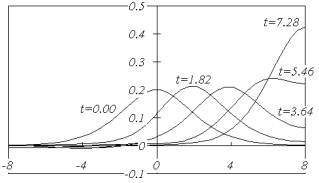

(a) Going to wall

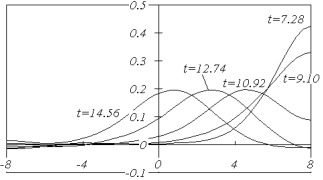

(b) Returning from wall
Fig.4 Propagation profiles of a solitary wave $(\operatorname{Re}=\infty)$

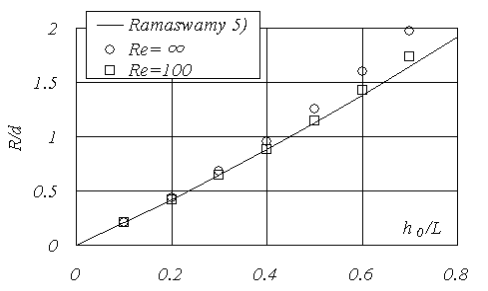

Fig.5 Run-up height of a solitary wave on a wall

\section{FLOW CALCULATION in VERTICAL CAVITY with FREE SURFACE and BOTTOM OPENING to WATER STREAM}

\subsection{Calculating conditions}

Fig. 6 shows the calculation model used for a vertical cavity with a free surface. The vertical cavities with free surfaces are located on the upper wall of a channel flow. Two vertical cavities are set on the upper wall. We expect that two free surface motions in the cavities will be generated in opposite directions, while the flowing water volume remains constant.

The following boundary conditions were used; the channel bottom and the vertical walls of the cavity are in slip conditions, the channel ceiling is in non-slip condition, and both inflow and outflow satisfy Direchlet condition $\left(\mathrm{U}=\mathrm{U}_{0}\right)$. Usually in the calculation of a channel flow, we use the Neumann condition for the boundary condition at the outflow part. However, if the outflow part is given with the Neumann condition in this case, the water will flow out toward the ground direction because of the effect of gravity. When the volume of inflow and outflow are not equal, the mean level of the free surface will vary. Therefore we set $U=U_{0}$ into the boundary condition of the outflow part.

The dimensionless governing equation, Eq.(1), has two parameters, namely the Reynolds number Re and the Froude number Fr. We simultaneously determined both numbers with only one variable of the flow speed $U$, by setting the cavity width to $\mathrm{L}=0.05 \mathrm{~m}$, kinetic viscosity to $v=10^{-5} \mathrm{~m}^{2} / \mathrm{sec}$, and the acceleration due to gravity to $\mathrm{g}=9.81 \mathrm{~m} / \mathrm{sec}^{2}$. The kinetic viscosity $v$ was set to about $1 / 10$ of that of water to ensure the stability of the calculation.

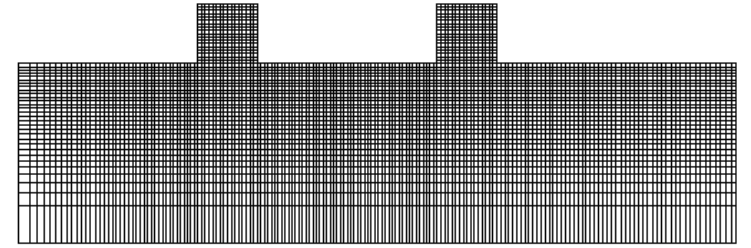

(a) Calculation mesh

(cavity: $16 \times 16$ grid, channel: $30 \times 178$ grid)

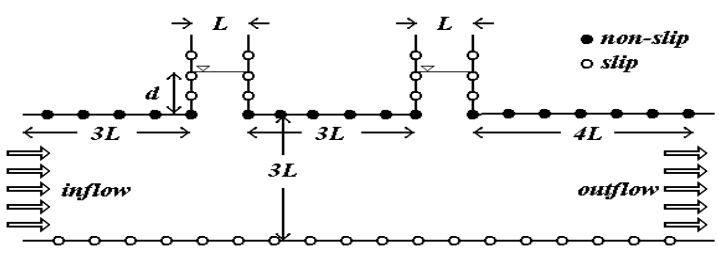

(b) B oundary conditions

Fig.6 Calculation field consisting of a channel with two cavities with free surface

4.2 Natural frequency of vertical water movement The water in the cavity with the free surface on the water stream moves up and down at a certain water stream speed.

The natural frequency was calculated to consider the vertical motion. The natural frequency was obtained by allowing the water to flow freely from the initial condition in which the water level of the front cavity was elevated $(\mathrm{h} / \mathrm{d}=+0.2)$ and that of back cavity was lowered $(\mathrm{h} / \mathrm{d}=-0.2)$ at the no stream speed condition. The calculated data are plotted in Fig.7 as non-dimensional circular frequency of $\omega^{\prime}=2 \pi f_{0} \sqrt{L g}$ together with experimental line of Ref.1). In this figure, the horizontal axes represents the cavity depth to width ratio, d/L. The present calculated data differ somewhat from the experimental data.

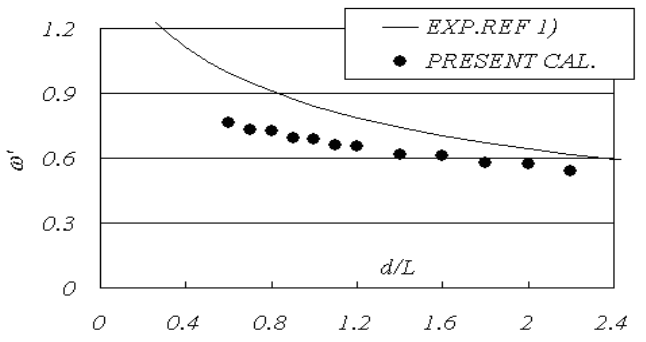

Fig.7 $\mathrm{N}$ atural circular frequency for vertical motion

4.3 Surface motion and flow in the cavity

In the numerical calculation, vertical motion of the free surface occurs at certain channel flow speeds. When the free surface of front cavity rises, the free surface of the rear cavity simultaneously goes down. The free surface motion is linked with the entering and leaving of vortexes in the cavity, as reported in Ref. 1). These phenomena for the highest and lowest free surface levels are shown in Fig.8. Fig.9 compares the calculated result in the front cavity $(d / L=1.0)$ with the flow visualized data from Ref. 1). 


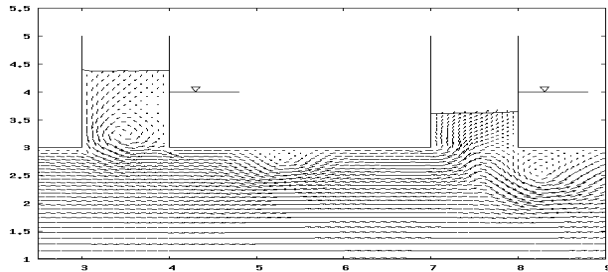

(a) at $\mathrm{t}=0.27 \mathrm{~T}_{0}$

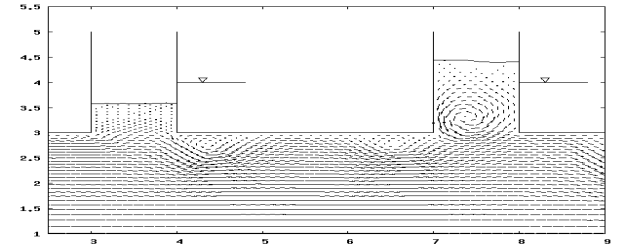

(b) at $\mathrm{t}=0.73 \mathrm{~T}_{0}$

Fig.8 Computed flow around two cavities ( $d / L=1.0, R e=1445, F r=0.41, T_{0}:$ cycle time )

(1) at $t=0.27 T_{0}$
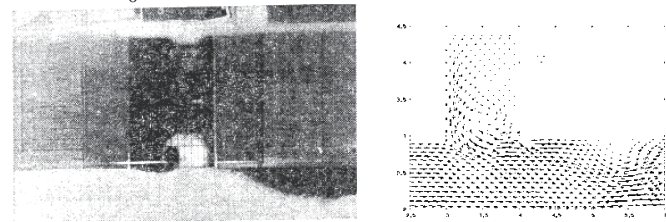

(2) at $t=0.73 T_{0}$
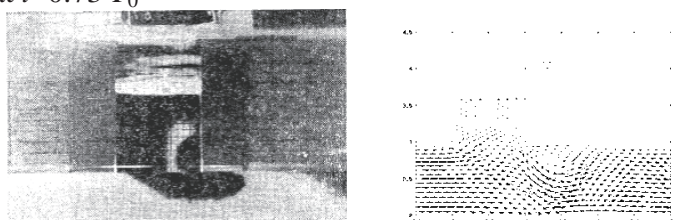

(a) Experiment in Ref. 1)

(b) Present Calculation ( $\mathrm{Re}=46800, \mathrm{Fr}=0.36$ )

( $\operatorname{Re}=1445, \quad F r=0.41$ )

Fig.9 Flow visualization and Calculation in cavity

Fig.10 shows the calculated results in the shallow cavity $(\mathrm{d} / \mathrm{L}=0.8)$ and the deep cavity $(\mathrm{d} / \mathrm{L}=2.0)$. When $\mathrm{d} / \mathrm{L}$ exceeds 0.6 , the vertical motion occurs. In the deep cavity, the vertical motion appears with almost flat condition of the free surface. However in the case of the shallow cavity, the vertical motion takes with the distorted free surface. When the depth of the cavity is shallow compared with the width, i.e. $d / L<0.5$, the water moves backward and forward as a sloshing motion in the tank. In that case, the calculation breaks down because the free surface reaches the channel ceiling. In this study, the depth to width ratio is constrained to lie between $0.8<\mathrm{d} / \mathrm{L}<2.0$, so that the free surface motion is mainly vertical.

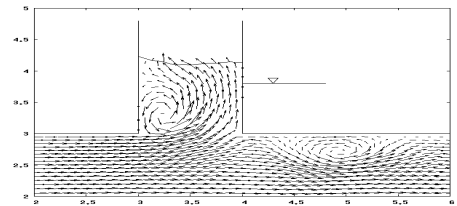

(a) Shallow Cavity ( $d / L=0.8)$

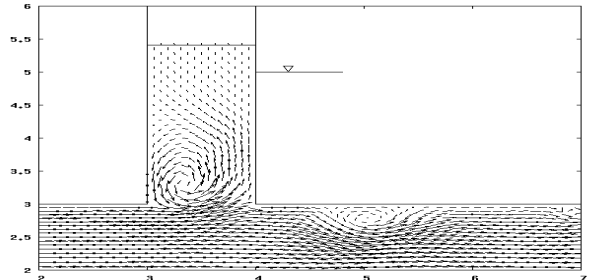

(b) Deep Cavity ( $\mathrm{d} / \mathrm{L}=2.0$ )

Fig. 10 Effect of cavity depth/width ratio (d/L)

4.4 Amplitude and frequency of the free surface motion

Fig.11 shows a comparison of the calculated amplitude and the experimental data ${ }^{1)}$. The horizontal axis is $2 U /\left(L \omega_{0}\right)$, where $U$ is the channel flow velocity, $L$ is the cavity width, $\omega_{0}$ is the natural circular frequency of the calculated and measured vertical motion in Fig.7. The value of $2 \mathrm{U} /\left(\mathrm{L} \omega_{0}\right)$ is the same as $1 /(\pi \mathrm{St})$, where St is the Strouhal number, $\mathrm{St}=\mathrm{f}_{0} \mathrm{~L} / \mathrm{U}$. It can be seen that the moving zone is the same in both the calculation and the experiment, approximately $0.6<2 \mathrm{U} /\left(\mathrm{L} \omega_{0}\right)<1.8$.

Fig.12 shows the time histories of up and down motion of the free surface in the cavity with dimensionless time. The up and down motion of the free surface occurs at the beginning of the calculation at the speed over $2 \mathrm{U} /\left(\mathrm{L} \omega_{0}\right)>0.6$. However the moving amplitude decays with time when the speed is $2 \mathrm{U} /\left(\mathrm{L} \omega_{0}\right)>1.8$.

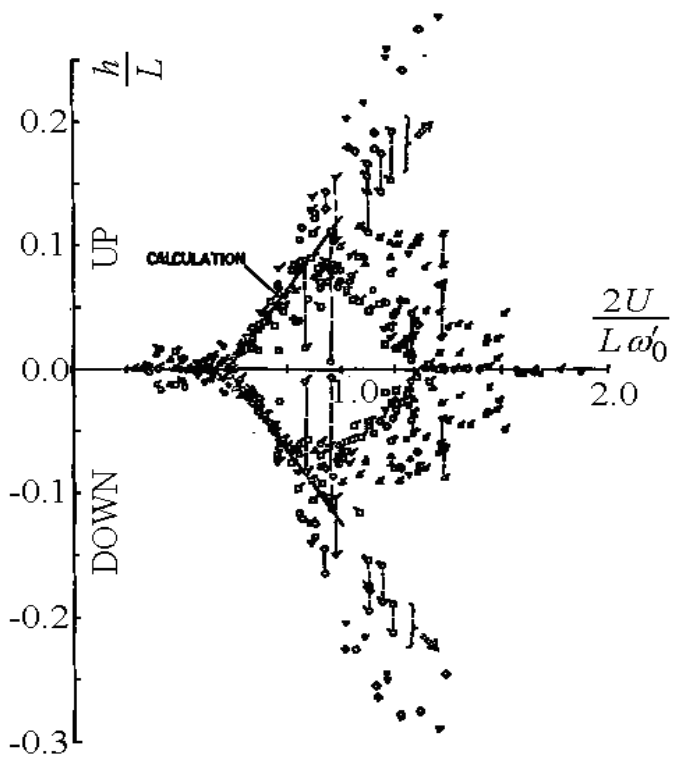

(a) Experiment in Ref. 1)

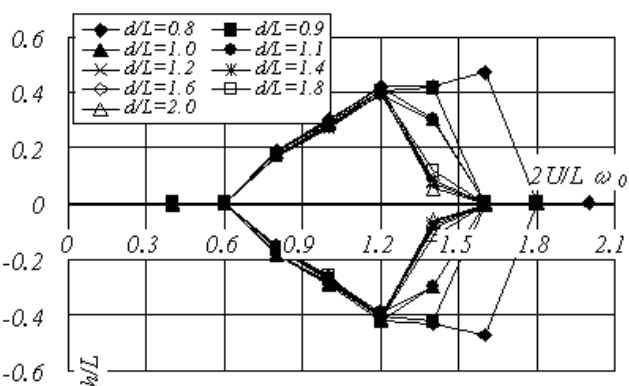

(b) Present Calculation

Fig.11 Up \& down moving amplitude of free surface 


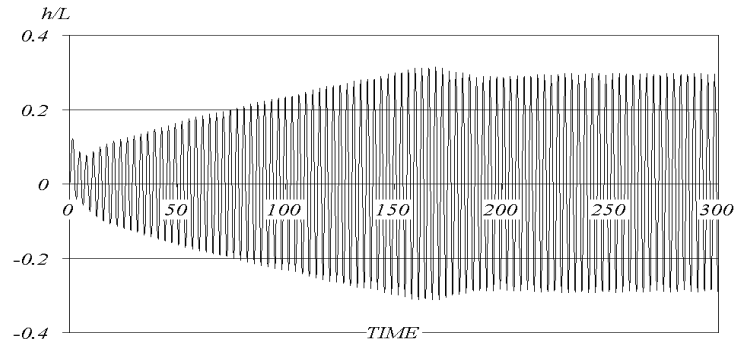

(a) M oving amplitude at $2 \mathrm{U} /\left(\mathrm{L} \omega_{0}\right)=1.0, \mathrm{~d} / \mathrm{L}=1.0$

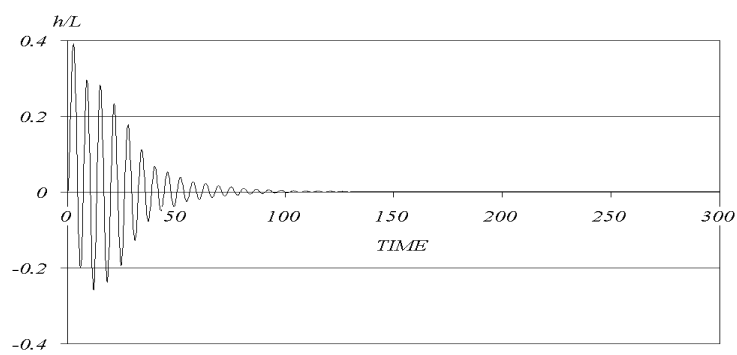

(b) M oving amplitude at $2 \mathrm{U} /\left(\mathrm{L} \omega_{0}\right)=2.0, \mathrm{~d} / \mathrm{L}=1.0$

Fig.12 Time histories of calculated free surface amplitude

The calculated moving amplitude is larger than that of the experiment. We suppose that the reason for this is the fact that the calculation was done with the two-dimensional cavities of the same size, whereas the experiment had been carried out with the three-dimensional ship models which had one cavity, moon pool, and the other was the large water surface surrounding the ship model.

Fig.13 shows the calculated and experimental circular frequencies of the vertical movement. The frequencies are normalized by the calculated and experimental natural frequencies. The figures agree well each other. The lock-in phenomena of the oscillating frequency of the free surface are observed in both the calculated and experimental data.

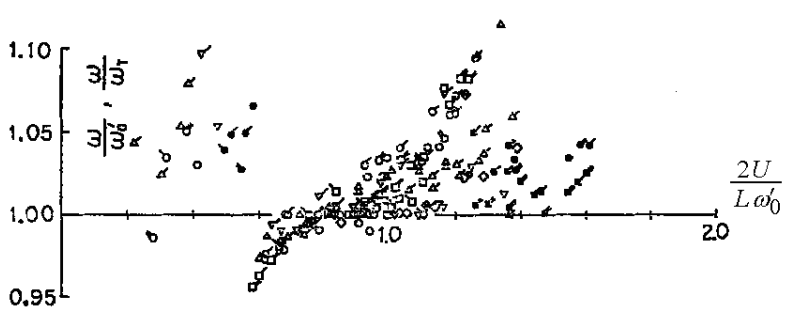

(a) Experiment in R ef. 1)

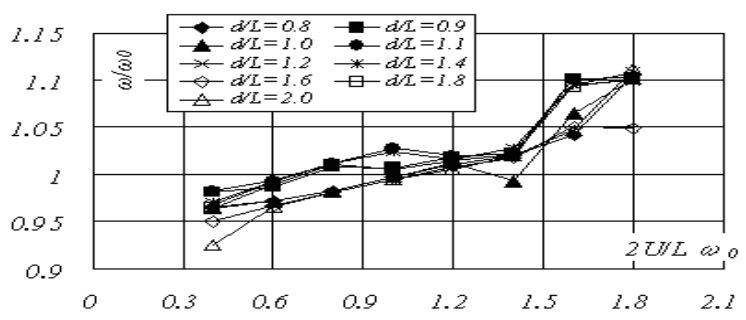

(b) Present calculation

Fig.13 M oving frequency (up and down motion)

\section{CONCLUSION}

In this study, the flow in the two-dimensional cavities with a free surface and a bottom opening to a water stream were numerically calculated using the GSMAC-FEM method. The ALE formulation was applied to adapt to the FEM mesh movement for the free surface motion. In order to confirm the accuracy of the calculation method three cases were examined; normal cavity flow, free oscillation motion in a tank and the run up height of a solitary wave on the tank wall. The calculated results were compared with the previously published data. A nd theses tested cases demonstrated that the present algorithm is accurate for the cavity flow calculation with a free surface.

Using the present calculation technique, the flow in a cavity with a free surface and a bottom opening to a channel flow was calculated and the following conclusions were obtained. (1) When two vertical cavities with a free surface and a bottom opening are set on the upper wall of a channel flow, the free surface moves up and down in the stream velocity range $0.6<$ $2 \mathrm{U} /\left(\mathrm{L} \omega_{0}\right)<1.8$. The value of $2 \mathrm{U} /\left(\mathrm{L} \omega_{0}\right)$ is the same as $1 /(\pi \mathrm{St})$, where $\mathrm{St}$ is the Strouhal number, $\mathrm{St}=\mathrm{f}_{0} \mathrm{~L} / \mathrm{U}$. These results agree well with the experimental data which were measured in the vertical openings of ship models.

(2) The dimensionless circular frequencies $\left(\omega / \omega_{0}\right)$ of the vertical motion agree well with the experimental data measured in the vertical openings of ship models. In both calculation and experiment results, the lock-in phenomena of the oscillation are observed.

\section{REFERENCES}

1) K. Fukuda: Behavior of water in Vertical W ell with Bottom Opening of Ship, Journal of the Society of Naval Architects of J apan, V ol.141, Page115-130, 1977

2) A. B. A albers: The $W$ ater M otions in a M oonpool, Ocean Engineering, Vol.11, No.6, pp. 557-579, 1984

3) G. Hashimoto and T.Tanahashi: Evaluation and Application of Higher-Order Elements to GSM A C-FEM, Transaction of JSCES, V ol.7, Paper N o. 20040030, page 149-157, 2005

4) U. Ghia, K. N. Ghia, C. T. Shin: High-Re Solutions for Incompressible Flow U sing the Navier-Stokes Equations and a Multigrid Method, Journal of Computational Physics, 48, 1982, pp. 387-411

5) B. Ramaswamy: Numerical Simulation of Unsteady Viscous Free Surface Flow, Journal of Computational Physics, 90, 1990, pp. 396-430

6) T. Nishimura, N. Kondo, M. Kano: N umerical A nalysis of Incompressible V iscous Flows with Free Surface, J. Struct. Constr. Eng. AlJ, N 0.490, 247-255, Dec, 1996 